\title{
$\mathrm{K}^{+}$Channels Expression in Hypertension After Arterial Injury, and Effect of Selective Kv1.3 Blockade with PAP-1 on Intimal Hyperplasia Formation
}

\author{
P. Cidad • L. Novensà • M. Garabito • M. Batlle • \\ A. P. Dantas • M. Heras • J. R. López-López • \\ M. T. Pérez-García $\cdot$ M. Roqué
}

Published online: 28 October 2014

(C) Springer Science+Business Media New York 2014

\begin{abstract}
Introduction $\mathrm{K}^{+}$channels are central to vascular pathophysiology. Previous results demonstrated that phenotypic modulation associates with a change in Kv1.3 to Kv1.5 expression, and that Kv1.3 blockade inhibits proliferation of VSMCs cultures.

Purpose To explore whether the Kv1.3 to Kv1.5 switch could be a marker of the increased risk of intimal hyperplasia in essential hypertension and whether systemic treatment with Kv1.3 blockers can prevent intimal hyperplasia after endoluminal lesion .

Methods Morphometric and immunohistochemical analysis were performed in arterial segments following arterial injury and constant infusion of the Kv1.3 blocker PAP-1 during 28 days. Differential expression of $\mathrm{K}^{+}$channel genes was studied in VSMC from hypertensive (BPH) and normotensive (BPN) mice, both in control and after endoluminal lesion. Finally, the migration and proliferation rate of BPN and BPH VSMCs was explored in vitro.

Results Changes in mRNA expression led to an increased $\mathrm{Kv} 1.3 / \mathrm{Kv} 1.5$ ratio in BPH VSMC. Consistent with this,
\end{abstract}

P. Cidad and L. Novensà contributed equally to the study.

P. Cidad · J. R. López-López • M. T. Pérez-García

Departamento de Bioquímica y Biología Molecular y Fisiología and Instituto de Biología y Genética Molecular (IBGM), Universidad de Valladolid and CSIC, Valladolid, Spain

L. Novensà • M. Garabito • M. Batlle • A. P. Dantas • M. Heras • M. Roqué

Cardiology Department, Institut del Tòrax, Hospital Clínic and Institut d'Investigacions Biomèdiques August Pi i Sunyer (IDIBAPS), University of Barcelona, Barcelona, Spain

\section{Roqué $(\bowtie)$}

Cardiology Department, Institut del Tòrax, Hospital Clínic de

Barcelona, Villarroel, 170, Barcelona 08036, Spain

e-mail:mroque@clinic.ub.es arterial injury in BPH mice induced a higher degree of luminal stenosis, ( $84 \pm 4 \%$ vs. $70 \pm 5 \%$ in BPN, $p<0.01)$, although no differences in migration and proliferation rate were observed in cultured VSMCs. The in vivo proliferative lesions were significantly decreased upon PAP-1 systemic infusion (18 \pm $6 \%$ vs. $58 \pm 20 \%$ with vehicle, $p<0.05$ ).

Conclusions Hypertension leads to a higher degree of luminal stenosis in our arterial injury model, that correlates with a decreased expression of Kv1.5 channels. Kv1.3 blockers decreased in vitro VSMCs proliferation, migration, and in vivo intimal hyperplasia formation, pointing to Kv1.3 channels as promising therapeutical targets against restenosis.

Keywords $\mathrm{K}^{+}$channels $\cdot$ PAP-1 $\cdot$ Intimal hyperplasia . Hypertension

\section{Introduction}

Vascular remodeling is a physiological response to altered blood flow conditions, changes in blood pressure, and atherosclerosis, and is a common finding in conditions such as hypertension and restenosis. Remodeling involves changes in VSMC growth and migration as well as alterations in the extracellular matrix composition.

Ion channels have a key role in the processes of cell proliferation and migration in the vessel wall, which are central to intimal hyperplasia formation, as well as in the maintenance of vascular tone, such as in hypertension. Among them, $\mathrm{K}^{+}$channels have been proposed as important determinants of contractile functional changes in hypertension [1] and in the phenotypic switch of vascular smooth muscle cells after arterial injury [2].

Hypertension, a well-recognized risk factor for cardiovascular disease and atherosclerosis development, has also been 
related to altered $\mathrm{K}^{+}$channel function. Whether hypertension by itself can have an impact on intimal hyperplasia formation after arterial injury is unknown because atherosclerosis development in humans is usually the consequence of many factors. A spontaneously hypertensive mouse model is available BPH (Blood Pressure High). Mean life span of these mice is reduced compared to BPN (Blood Pressure Normal) mice (20 versus 28 months, respectively), reflecting the shortened life span related to hypertension complications in these animals [3].

$\mathrm{K}^{+}$channels are the therapeutic target of a number of drugs and are, therefore, suitable to be modulated in a clinical setting. Recent investigations in the $\mathrm{Kv}$ channel field are advancing in the development of new compounds to target $\mathrm{Kv}$ channels for several conditions, such as autoimmune diseases, multiple sclerosis, epilepsy, cancer, and cardiovascular disorders [4].

In our previous work, using high-throughput real-time PCR, we obtained a global portrait of ion channel expression from contractile and proliferative VSMCs obtained from mice femoral arteries in two experimental paradigms: an in vivo model of endoluminal lesion and an in vitro model of cultured VSMCs obtained from explants. We observed a general decrease of ion channels expression upon proliferative switch, except for the voltage-dependent potassium channel (Kv) $\mathrm{Kv} 1.3$ and the $\mathrm{Kv} \beta 2$ auxiliary subunit. Kv1.3 functional expression associates to proliferative phenotype, as we also found an in vitro inhibitory effect of the Kv1.3 selective blockers margatoxin (MgTx, a venom toxin) and the psoralen PAP-1 on VSMCs proliferation and migration [2]. However, not such a study has been performed in the in vivo model of endoluminal lesion.

Although there are several other ion channels (including TRPC1) [5], T-type calcium channels [6], Kv3.4 channels [7, $8]$ and IK1 channels $[9,10]$ whose expression and/or function has been linked to VSMC proliferation, the information regarding conservation across different vascular beds and/or species is missing for most of them. Besides, involvement of Kv1.3 channels in proliferation has been described in a substantial number of cell types, including T- and B-cells, macrophages, platelets, endothelial cells, microglia, adipocytes, oligodendrocyte progenitors and some cancer cells $[4,11,12]$.

In proliferating mouse femoral VSMCs, increase of Kv1.3 currents (determined by electrophysiological recordings in isolated cells) parallels an enormous decrease of Kv1.5, the dominant $\mathrm{Kv} 1$ channel in the contractile state, and following this observation, we found that the Kv1.3 to Kv1.5 ratio was a landmark of the VSMC phenotype in all vascular beds studied [13]. Moreover, Kv1.3 expression was also able to induce proliferation when heterologously expressed in HEK cells, while Kv1.5 expression inhibits it. This antagonistic effect of Kv1.5 and Kv1.3 channels in this system points to concurrent changes in Kv1.5 and Kv1.3 channels expression in native systems as a requirement for the functional changes associated with phenotypic switch [13].

In the present study, we sought to address two main issues: first, whether hypertension, in the absence of other risk factors, has an impact in the response-to-injury. We will also explore if the differences in the in vivo proliferative response in our arterial injury model correlate (and hence could be predicted) by the remodeling of $\mathrm{Kv}$ channels, and more specifically by a change in the Kv1.3 to Kv1.5 ratio that could promote a pro-proliferative state under these conditions. Second, we will explore the effect of the specific blockade of Kv1.3 channels on in vivo intimal hyperplasia formation.

\section{Materials and Methods}

Animals

BPN strain mice were purchased from Jackson Laboratories (Bar Harbor, Maine, USA) and maintained with inbred crossing in the animal facility of the Hospital Clínic of Barcelona School of Medicine. Animals were housed under temperaturecontrolled conditions $\left(21^{\circ} \mathrm{C}\right)$ with free access to water and food.

All animal procedures have been approved by the University of Barcelona Ethics Committee on Animal Experimentation.

$\mathrm{BPH}$ mice have a hypertension level qualified as moderate. BPH mice have a mean systolic and diastolic BP of $153 \pm$ $3 \mathrm{mmHg}$ and $107 \pm 2$, respectively; compared with BPN mice (sBP of $135 \pm 2 \mathrm{mmHg}$ and $\mathrm{dBP}$ of $90 \pm 2 \mathrm{mmHg}$.

Surgical Procedures and in-vivo PAP-1 Treatment For transluminal injury of femoral arteries, endoluminal lesion to the common femoral artery was achieved as previously described [14]. In this model, we also explored the effects of treatment with the selective Kv1.3 blocker PAP-1 [15] (Sigma-Aldrich, Germany). For that purpose, BPN animals, underwent endoluminal injury 2 days after subcutaneous minipump (Alzet Corp.) implantation, so that a constant systemic delivery of PAP-1 (or vehicle) could be obtained. PAP-1 was administered at $50 \mu \mathrm{g} / \mathrm{kg} / \mathrm{min}$ during up to 28 days after femoral artery injury. Control groups were injured animals infused with vehicle (Cremophor C5135, Sigma-Aldrich, Germany), and sham-operated animals infused with PAP-1. Femoral arteries were collected and cross-sections from all the experimental groups were stained and analyzed morphometrically.

\section{Proliferation and Migration Assays}

BPN and BPH mice from 4 to 6 weeks of age were euthanized and the femoral arteries were carefully dissected. After 
cleaning of connective and endothelial tissues, primary cultures of VSMCs were obtained as previously described $[2,8]$.

Femoral cultured VSMCs from BPN and BPH mice were plated in poli-L-lysine coated coverslips and grown to approximately $65-80 \%$ confluence. At this point, cells were synchronized by $48 \mathrm{~h}$ incubation in SF (serum-free) medium at $37^{\circ} \mathrm{C}$ in a $5 \% \mathrm{CO} 2$ humidified atmosphere. After this, cells were incubated in the indicated experimental conditions for $24 \mathrm{~h}$ and proliferation was determined by BrdU incubation for an additional $60 \mathrm{~min}$ period as previously described [2] . BrdU incorporation was measured and represented as the percentage of BrdU positive cells (BrdU+) from the total cell number stained with DAPI. In each experiment, BrdU incorporation was obtained from the average of at least 10 different fields for each condition.

To evaluate migration we used a wound healing or scratch assay in cultured VSMCs from mouse femoral arteries [2]. The scratched area was measured using Image $\mathrm{J}$ software. Percentage of invaded area was determined as \% Reinvasion, according to the following expression:

$\%$ Reinvasion $=100($ Area0 - Area24 $) /$ Area0

Where Area $0=$ Initial area $(t=0)$ and Area24=area at $t=$ $24 \mathrm{~h}$ after scratch. Migration assays were performed in VSMCs derived from BPN or BPH arteries both in the absence (control) or presence of $100 \mathrm{nM}$ PAP-1.

\section{Histology and Morphometry}

For the morphometrical analysis, animals were euthanized 48 h, 1 and 4 weeks after arterial injury and perfusion-fixed with $4 \%$ paraformaldehyde in PBS for $5 \mathrm{~min}$ at $100 \mathrm{mmHg}$ to obtain paraffin sections from femoral arteries as described elsewhere $[2,14]$. Three 5 - $\mu$ m-thick paraffin sections from each arterial segment were stained with combined Masson's trichrome elastic and analyzed by using computerized morphometry (NIH Image) and the average values were calculated. Measurements included luminal area, medial area, intimal area, vessel area, and the lengths of the internal elastic lamina and external elastic lamina. The intima-to-media (I/M) ratio and the percentage of stenosis were calculated as described [14].

Immunohistochemistry

Immunohistochemical analysis was performed as previously described [2]. Briefly, arterial sections were treated with $1 \%$ citric acid for antigen retrieval and blocked with $3 \%$ hydrogen peroxide (DakoCytomatation) before the incubation with primary antibodies at $4{ }^{\circ} \mathrm{C} \mathrm{ON}$. Sections were incubated with a horseradish peroxidase conjugated anti-mouse IgG
(DakoCytomatation) for $30 \mathrm{~min}$ and signals were detected using 3,3-diaminobenzidine (DAB, DakoCytomatation). Negative controls were prepared by substitution of the primary antibody with an irrelevant antibody. Primary antibodies (prepared at 1:50) were: mouse anti-Kv1.3 (clone L23/27, NeuroMab, UC Davis), mouse anti-Kv1.5 (Sigma) mouse anti-Kvbeta2 (Santa Cruz Biotech), rabbit anti-TWIK1 and rabbit anti-BKCa Alomone Labs. For immunohistochemical measurements four magnified $(\times 20)$ areas of the vessel wall, acquired from each of the four quadrants of the arterial circumference, were blindly analyzed (Image AnalySIS 2.3). The percentage of labelled area over the total vessel area was calculated for all arteries.

\section{RNA Expression Profile}

Taqman low-density arrays (Applied Biosystems) were used to simultaneously quantify the expression of 96 genes/sample as previously described [2, 13, 16]. These genes include $\alpha, \beta$ and $\gamma$ subunits of $\mathrm{K}^{+}$channels as well as $\alpha$ subunits of voltage-dependent $\mathrm{Ca}^{2+}$ channels (VDCC), $\mathrm{Cl}^{-}$channels and TRP channels. The expression levels of markers of VSMCs (calponin, Cnn1), and endothelium (endothelial nitric oxide synthase, NOs3, and Von Willebrand factor, VWf), were also explored, as well as other endogenous controls (Gapdh, B2m, Hprt-1 and Klf5). Each sample derived from $\approx 500 \mathrm{ng}$ of mRNA from contractile or proliferating VSMCs (610 femoral arteries for each determination). Briefly, total RNA from arteries was isolated with MELT ${ }^{\mathrm{TM}}$ Total RNA Isolation System Kit (Ambion). Real-time PCR with TaqMan Low Density Arrays (Applied Biosystems) was carried out with the ABI Prism 7900HT Sequence detection system (Applied Biosystems). mRNA expression levels were determined using the threshold cycle (Ct) relative quantification method $(\Delta \Delta \mathrm{Ct})$ [17]. Expression data of genes were normalized by an internal control, ribosomal RNA 18S, Gapdh or Gusb. The relative abundance of the genes was calculated from $2^{(-\Delta \mathrm{Ct})}$, where $\Delta \mathrm{Ct}=\mathrm{Ctgen-Ctinternal}$ control.

Changes in the expression between control (calibrator) and the different experimental conditions were calculated from

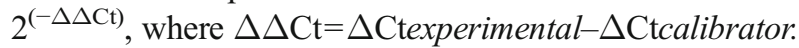

Statistical Analysis

A number of 15 animals per group to reach statistical significance has been calculated, considering the loss of some animals because of accidental death.

To compare different groups an analysis of variance (ANOVA) and non-paired $t$-test have been applied. Values are provided as Means \pm SEM. 


\section{Results}

Effects of Hypertension on Intimal Hyperplasia After Arterial Injury

We selected BPN and BPH animals having similar ages and body weights, to ensure homogeneity of femoral artery size. A gender analysis was performed, that did not find any significant differences between males and females in all subgroups and time-points analyzed (data not shown); therefore data from all animals has been pooled.

Forty-eight hours after injury, there was no detectable proliferative response in the intima layer of injured arteries, but a strong inflammatory response was observed, with deposition of blood-borne cells on the denuded luminal surface (Fig. 1a). One week after injury, a mild intimal hyperplasia was observed, without significant differences between BPN and BPH mice, (Fig. 1b). A robust proliferative response was observed in BPN and BPH mice 28 days after arterial injury (Fig. 1c), with a mean intima/media ratio of $1.8 \pm 0.3$ and $1.7 \pm$ 0.3 , respectively, $p=\mathrm{NS}$. Data is summarized in Table 1 .
However, despite a similar age and body weight of the animals in both groups, a significantly higher vascular area and luminal area was noted in BPN mice, compared with BPH (Table 1). Medial and intimal areas were comparable. This resulted in a significant increase in the percentage of luminal stenosis in BPH mice versus BPN. These morphometric data suggest a negative remodeling after arterial injury in BPH mice, that could be related to the increased vascular tone characteristic of hypertension, which translates in an increased degree of luminal stenosis.

Expression Profile of ion Channels in BPH Femoral VSMCs and Effect of Endoluminal Injury

In light of the previous findings, we explored whether changes in the expression profile of ion channels could help in defining risk factor conditions in the development of intimal hyperplastic lesions, and in particular the case of essential hypertension. We first determined the ion channel expression profile of femoral artery VSMCs from BPN and BPH mice, and after
Fig. 1 Intimal hyperplasia in BPN and BPH mice 48 h, 1 week, and 28 days after arterial injury. Representative microphotographs of femoral arteries from BPN (left panels) and BPH (right panels) mice, $48 \mathrm{~h}$ (A panels), 1 week (B panels) and 28 days (C panels) after injury a

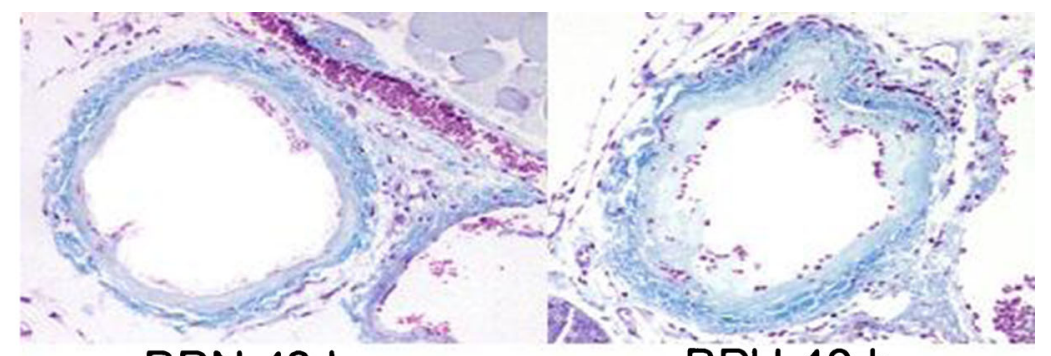
BPN $48 \mathrm{~h}$

b

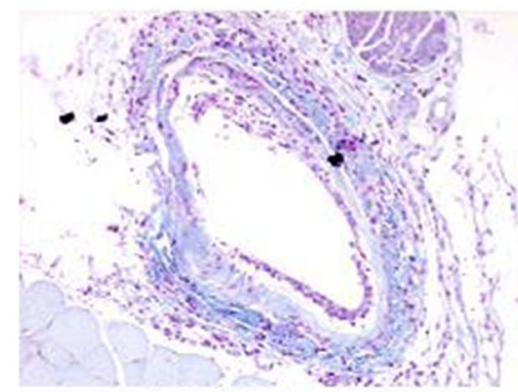

BPN $1 \mathrm{w}$

C

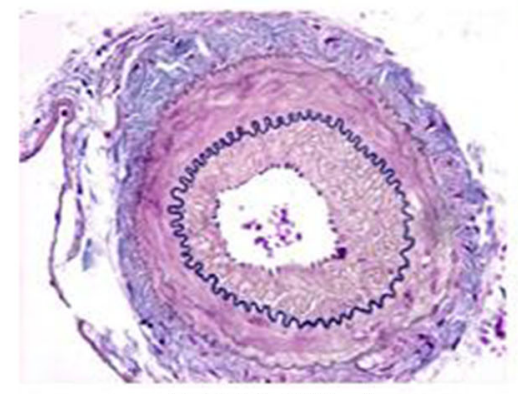

BPN $28 d$
$\mathrm{BPH} 48 \mathrm{~h}$

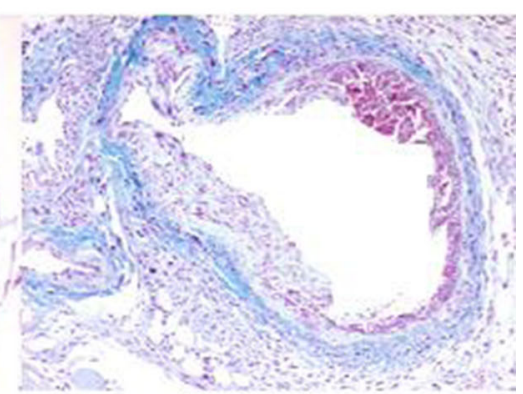

$\mathrm{BPH} 1 \mathrm{w}$

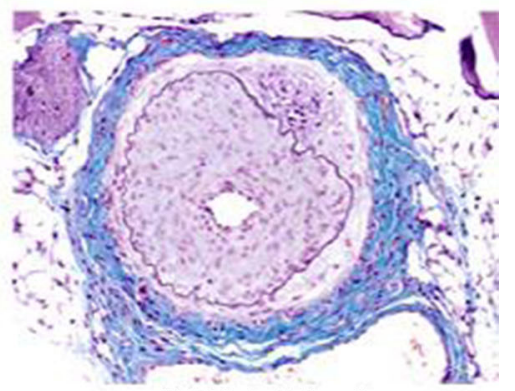

$\mathrm{BPH} 28 \mathrm{~d}$ 
Table 1 Morphometry of injured arteries from BPH and BPN mice 28 days after injury

\begin{tabular}{llllll}
\hline & Vascular area & Media area & Intima area & Luminal area & I/M ratio \\
\hline BPN $n=37$ & $38015 \pm 3717$ & $16824 \pm 2247$ & $17871 \pm 2017$ & $3320 \pm 884$ & $1.7 \pm 0.3$ \\
BPN $n=24$ & $51769 \pm 4696^{*}$ & $19178 \pm 2907$ & $24073 \pm 3544$ & $8888 \pm 2204^{* *}$ & $1.8 \pm 0.3$ \\
\hline
\end{tabular}

I/M ratio intima/media ratio

${ }^{*} p<0.05 ;{ }^{* *} p<0.01$

that we explored the changes in this expression profile upon endoluminal injury in BPH mice.

Expression of 31 channel genes was undetectable after 40 cycles of amplification in all conditions. The relative abundance of the 49 channel genes found in the control preparation, (mRNA from intact BPN femoral arteries without endothelium), is shown in Fig. 2a. Genes are grouped by families, and their expression levels normalized to the endogenous control ribosomal protein $18 \mathrm{~S}$.

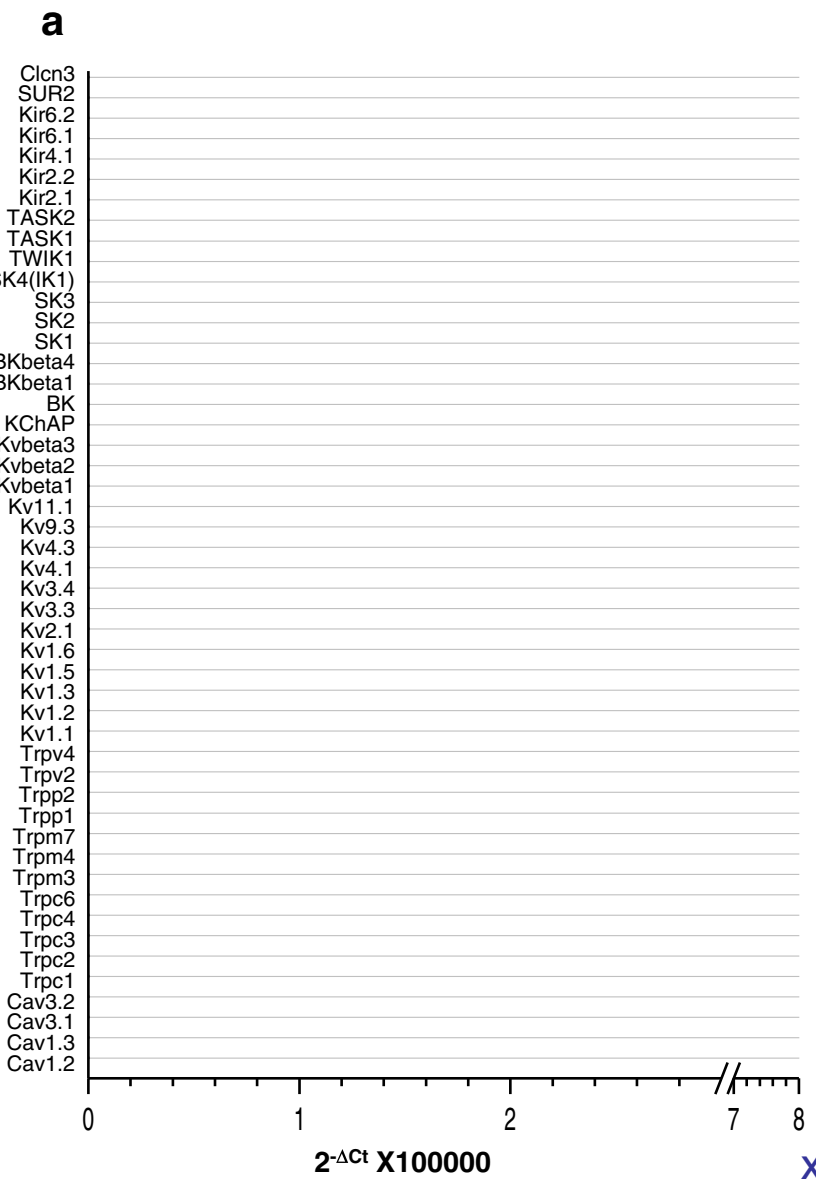

Fig. 2 a Relative abundance of the ion channels expressed in femoral VSMCs from BPN mice. Expression levels are normalized with respect to RP18s. Relative abundance was expressed as $2^{-\Delta \mathrm{Ct}} \times 10^{5}$, where $\Delta \mathrm{Ct}=$ $\mathrm{Ct}_{\text {channel }}-\mathrm{Ct}_{18 \mathrm{~s}}$. The different families of ion channels are plotted with the same color, from top to bottom $\mathrm{Cl}$ channels ( $g r e y$ ) inward-rectifier channels (orange), two-pore $\mathrm{K}$ channels (dark green), Ca-dependent $\mathrm{K}$ channels (yellow) accessory subunits of Kv channels (dark blue), Kv channels (blue), TRP channels (green) and Ca channels (red). b Differences in ion
Changes in the expression profile of ion channels upon hypertension were studied by analyzing for each individual gene the differences between control (BPN) and BPH with the $2^{(-\Delta \Delta \mathrm{Ct})}$ relative quantification method (Fig. 2b), where $\Delta \Delta \mathrm{Ct}=\Delta \mathrm{Ct}_{\mathrm{BPH}}-\Delta \mathrm{Ct}_{\mathrm{BPN}}$ and $2^{(-\Delta \Delta \mathrm{Ct})}$ represents the expression fold change.

As shown in the figure, only one channel gene (TWIK1) shows a remarkable increase in expression in BPH mice, while there are several channel genes showing a significant

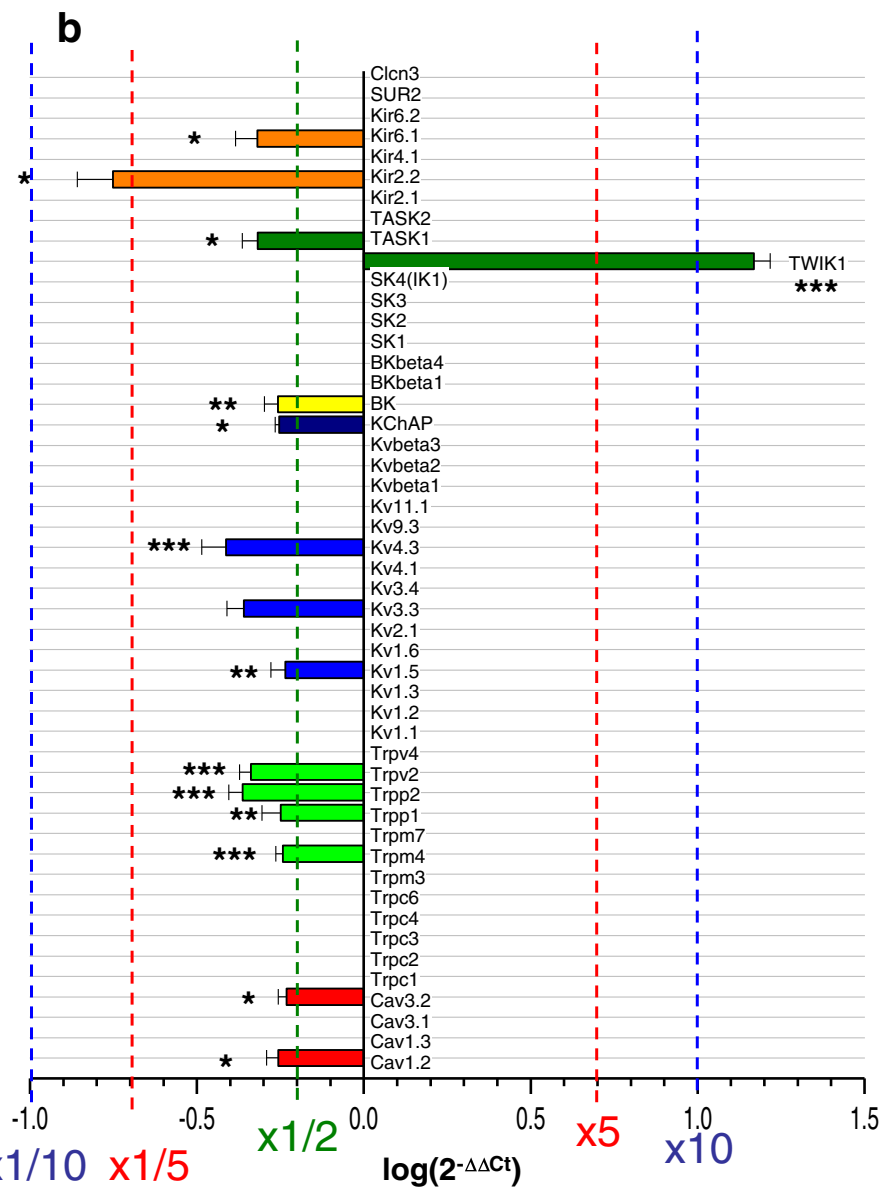

channels expression in BPH femoral arteries (using BPN expression levels as calibrator). The changes were calculated as $2^{-\Delta \Delta \mathrm{Ct}}$, where $\Delta \Delta \mathrm{Ct}=\Delta \mathrm{Ct}_{(\mathrm{BPH})}-\Delta \mathrm{Ct}_{(\mathrm{BPN})}$, and represented in log scale, so that negative values mean decreased expression and positive values increased expression. Each bar is the mean of 10 determinations obtained in 5 assays by duplicate. All through the figures, ${ }^{*} p<0.05,{ }^{* *} p<0.01$ and $* * * p<0.001$ 
decreased expression. These include some calcium channel genes (Cav1.2 and Cav3.2), several members of the TRP channel family, some inward rectifier channels (Kir6.1 and Kir2.2), the maxi-K channel (BK), a two pore domain channel (TASK1), the accessory subunit KChAP and several members of the Kv channel family (Kv4.3, Kv3.3 and Kv1.5).

Next we explored the changes between BPN and BPH femoral VSMCs when endoluminal injury was performed. In this case, the mRNA expression profile was obtained from injured BPN and BPH arteries extracted 28 days after surgery, and the expression levels obtained in BPN injured arteries were used as the calibrator, so that the changes will reflect the effect of hypertension on the phenotypic modulation. When compared with injured BPH arteries, we found changes in 15 channel genes, of which again only TWIK1 expression increased (Fig. 3a). Some of the channels that show a decreased expression are coincident with those that are down-regulated in hypertensive arteries. Of those, we found specially relevant the decreased expression of Kv1.5 in both situations, because, according to our previous work, VSMCs phenotypic switch associates with an increase in the Kv1.3 to Kv1.5 ratio [13]. While we did not observe changes in the expression of Kv1.3, which increased to the same levels as in injured BPN arteries [2], Kv1.5 expression was significantly decreased in injured
$\mathrm{BPH}$, leading to a higher Kv1.3 to Kv1.5 ratio (Fig. 3b) that is in agreement with the increased intimal thickening observed in response to injury in BPH VSMCs.

Ion Channels Protein Expression After Arterial Injury in BPH Versus BPN Mice

We explored the correlation and the protein level of some of the changes observed in our mRNA expression profile. We chose to explore the most conspicuous changes (i.e. the increased expression of TWIK1 channels in BPH) as well as some of the channels that have been previously associated with VSMC phenotypic modulation (such as, Kv1.3, Kv1.5 and $\mathrm{Kv} \beta 2$ ) or with hypertension (BK channels). For all these channel proteins, we analyzed their expression at three different times after endoluminal lesion (2, 7 and 28 days) on BPN and BPH arteries, using immunohistochemical techniques. Our findings are summarized in Fig. 4.

We did not observe consistent changes when comparing the expression levels of the channel proteins studied at $48 \mathrm{~h}$ and 7 days. However, the low rate of proliferative lesion together with the acute inflammatory response at these time points precludes any interpretation of the data. At 28 days after injury, a significant decrease of Kv1.5 expression in the a

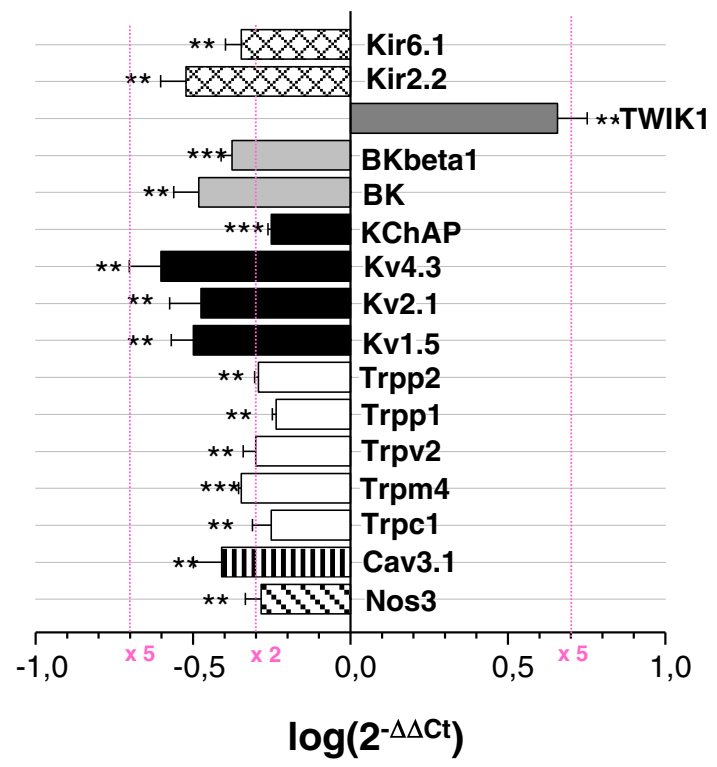

Fig. 3 a Bar plot showing the changes in the expression of ion channels in injured femoral arteries from BPH mice using injured BPN arteries as the calibrator. The plot calculations are made as in Fig. 2b. Only ion channels exhibiting a significant change in expression are represented, and as in the previous figure, negative and positive values indicated decreased and increased expression respectively. Data were obtained from injured arteries after 28 days of lesion, and the plot shows the mean \pm SEM of 8 determinations from 4 different experiments in duplicate determinations. b The relative abundance of Kv1.3 and Kv1.5 in BPN

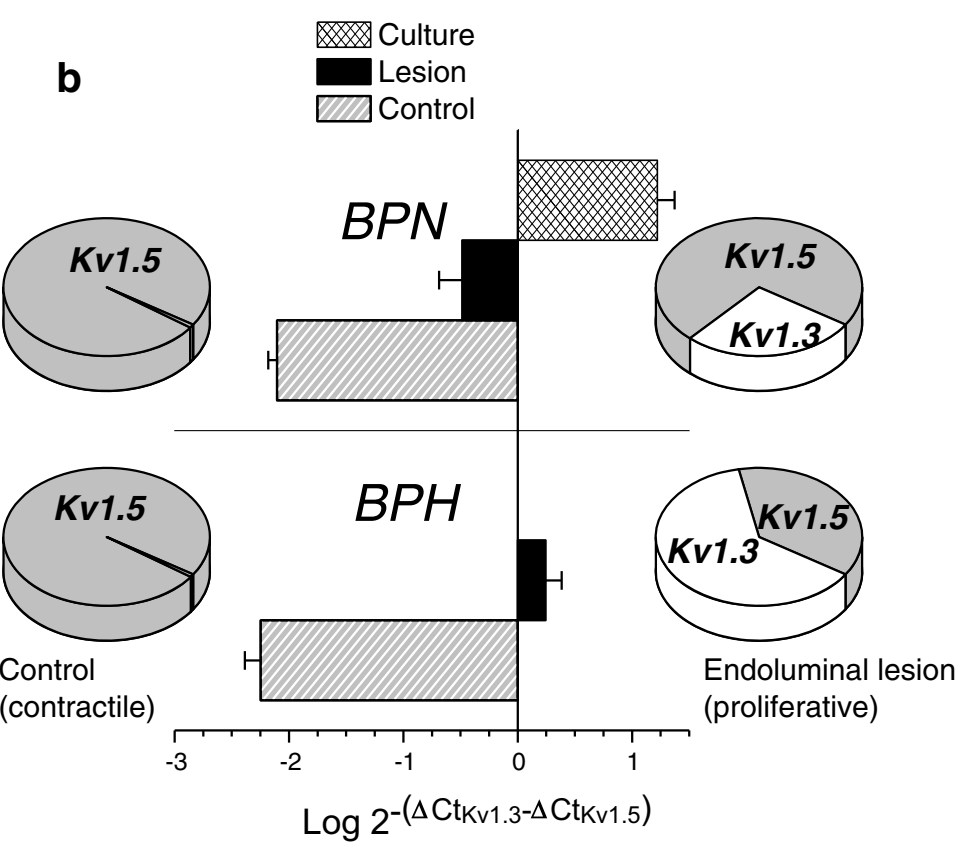

and BPH femoral VSMCs in both conditions (control and 28-days post lesion, injured) is illustrated by the pie charts. The bars plot shows the $\mathrm{Kv} 1.3: \mathrm{Kv} 1.5$ ratio expressed as $\log \left(2^{-\Delta \Delta} \mathrm{Ct}\right)$, where $\Delta \Delta \mathrm{Ct}$ was $\Delta \mathrm{CtKv1.3-} \Delta \mathrm{CtKv1.5}$. A value of 0 indicates a $\mathrm{Kv1.3:Kv1.5}$ ratio of 1 , a value of -2 denotes Kv1.5 expression levels 100 times higher than $\mathrm{Kv1} .3$, and a value of $+2 \mathrm{Kv} 1.3$ expression levels 100 times higher than Kv1.5. Each data point was obtained from at least 5 independent determinations. For comparison, the $\mathrm{Kv} 1.3: \mathrm{Kv} 1.5$ ratio obtained in primary cultures from femoral BPN VSMCs is also shown in the plot 
Fig. 4 Immunohistochemical expression of ion channels in the femoral artery of BPH and BPN mice 28 days after injury. a Immunohistochemistry for TWIK2, Kv1.3, Kv1.5, Kvb2 and $\mathrm{BKCa}$ in paraffin-embedded femoral artery cross-sections from $\mathrm{BPH}$ and BPN mice. Data are expressed as percentage of labelled area within the vessel wall. b Representative microphotographs of immunohistochemical expression of TWIK2 and Kv1.5 in femoral arteries of BPH and BPN mice 28 days after arterial injury a
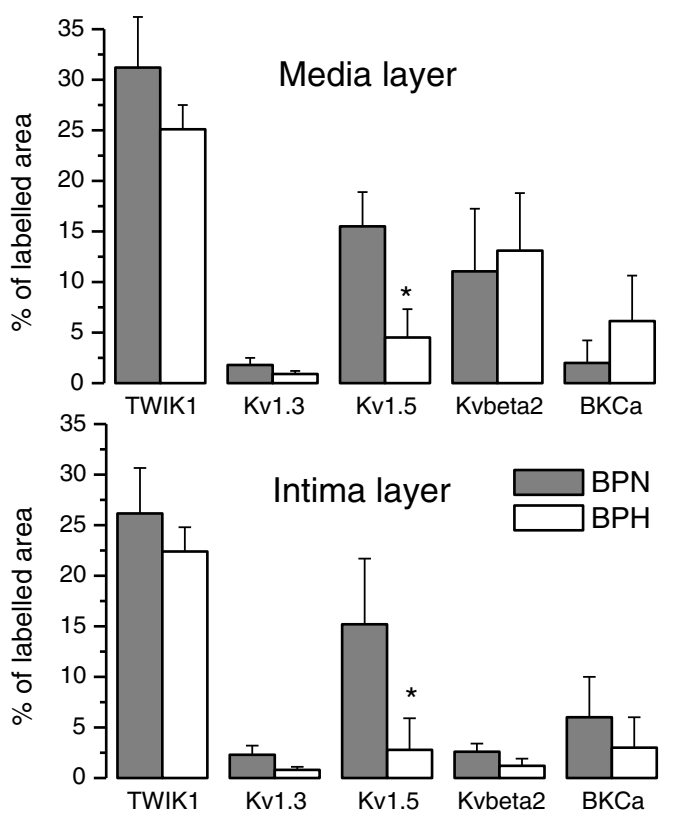

b

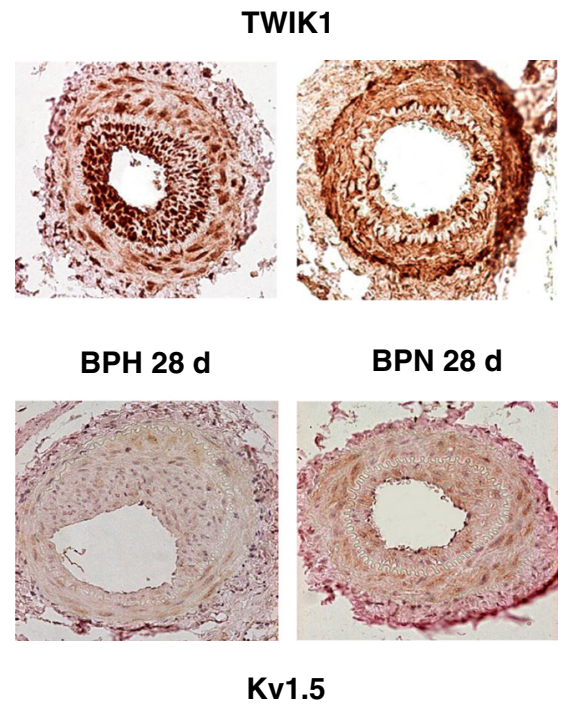

vessel wall of BPH arteries was observed. both in the media an in the intima layer. (Fig. 4a and b) There were no other major significant differences in vessel wall channel expression, as assessed by immunohistochemical analysis, between BPH and BPN mice. In the case of TWIK1, while the labelled area was large as expected, no significant differences could be observed when comparing BPN and BPH samples. Representative images of the labelling with TWIK1 and Kv1.5 antibodies in both preparations are shown in Fig. 4b.

\section{Proliferation and Migration of VSMCs from BPN and BPH} Arteries in vitro

Finally, we investigated if the changes in the proliferative response between BPN and BPH VSMCs observed in the in vivo model of proliferation could be also observed in vitro, by analyzing the rate of proliferation of primary VSMCs cultures (Fig. 5a). No differences in serum-induced proliferation between BPN and BPH were detected, which suggest that the increased proliferation of VSMCs from BPH mice is dependent on some environmental cues that, after several passages could be lost in the in vitro culture conditions. The inhibitory effect of 10nM MgTx, a selective Kv1.3 blocker, was also similar in both strains. We also determined that this $24 \mathrm{~h}$ treatment with $10 \mathrm{nM}$ MgTx did not modify the expression levels of Kv1.3 or Kv1.5 mRNA in cultured VSMCs (Fig. 5b).

We finally explored the possible differences in the migration rate of BPN and BPH VSMC in culture using a scratch assay. In this set of experiments, we evaluated both the migration rate of the cells in control media, as well as the effect of Kv1.3 blockade using $100 \mathrm{nM}$ PAP-1. As shown in Fig. 6, no significant differences in the migration rate could be observed between BPN and BPH femoral VSMCs cultures. Also, the inhibitory effect of treatment with PAP-1 was of similar magnitude in both cases, suggesting a similar contribution of Kv1.3 channels to migration in BPN and BPH cultures.

Effect of Selective Blockade of Kv1.3 on the in vivo Proliferative Response

We have previously described that Kv1.3 channels are upregulated in proliferating VSMCs both in vivo (endoluminal injury) and in vitro (cultured cells). In this later system we have also found that selective Kv1.3 blockers were able to inhibit proliferation. However, this point had not been explored in vivo, as well as the feasibility of a systemic treatment with Kv1.3 blockers. No changes in the morphometry of the arteries were observed upon PAP-1 treatment in the absence of arterial injury (Fig. 7a). A marked decrease in intima-to-media ratio in femoral arteries of animals treated with PAP-1 was observed, compared to vehicle-treated animals $(0.43 \pm 0.2$ versus $1.06 \pm 0.4$, respectively; $p<0.05$ ). This decreased intimal thickening translated also in a significant reduction in the percentage of luminal stenosis ( $18 \pm 6 \%$ versus $58 \pm 20 \%$, respectively; $p<0.05$; Fig. $7 \mathrm{a}$ and $\mathrm{b}$ ).

\section{Discussion}

We have used a well-characterized arterial injury model, developed by our group [14], that leads to reproducible intimal 
a

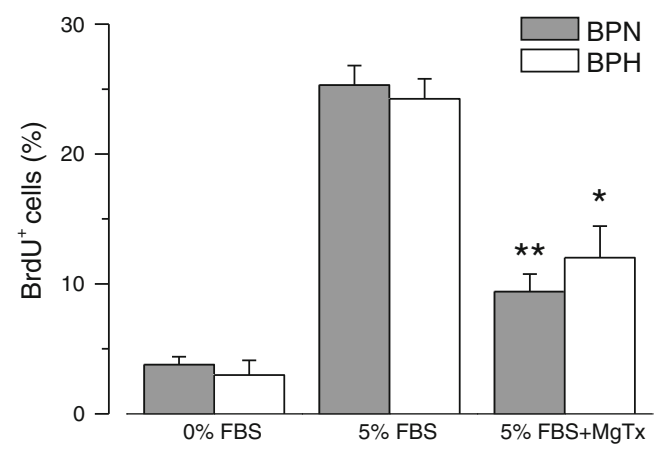

Fig. 5 a Proliferation rate of BPN and BPH VSMC and effect of Kv1.3 blockade. Proliferation rate of VSMCs from femoral artery BPH and BPN was determined as the percentage of cell incorporating BrdU after $24 \mathrm{~h}$ treatment with the indicated conditions. The bar plot shows the proliferation rate in the absence or in the presence of FBS (5\%) and the effect of the treatment with Margatoxin $(\mathrm{MgTx}, 10 \mathrm{nM})$ on the FBS induced proliferation. Experiments were carried out in parallel in BPN

hyperplasia, as happens after percutaneous coronary interventions and stent implantation. By using this injury model in mice with a similar genetic background, that have been phenotypically selected by their blood pressure levels as normotensive (BPN) or hypertensive (BPH), the present study provides some relevant conclusions. First, using BPN mice, we found that isolated essential hypertension leads to a higher degree of luminal stenosis in our arterial injury model. Second, we demonstrate that selective blockade of Kv1.3 channels with PAP-1 is an effective strategy to inhibit intimal hyperplasia after vascular interventions, as there was a very significant decrease in the proliferative response in the animals subjected to a chronic systemic administration of PAP-1.

a
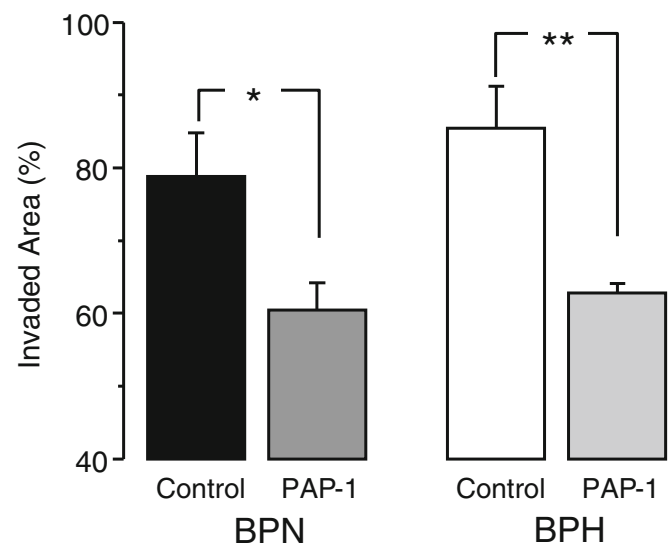

Fig. 6 Migration rate of BPN and BPH VSMC and effect of PAP-1. a Effect of Kv1.3 blockers on femoral VSMC migration calculated as the percentage of invaded area in scratch assays. Parallel experiments were carried out in femoral VSMCs obtained from BPN and BPH arteries. In both groups migration rate was determined in control conditions and in b

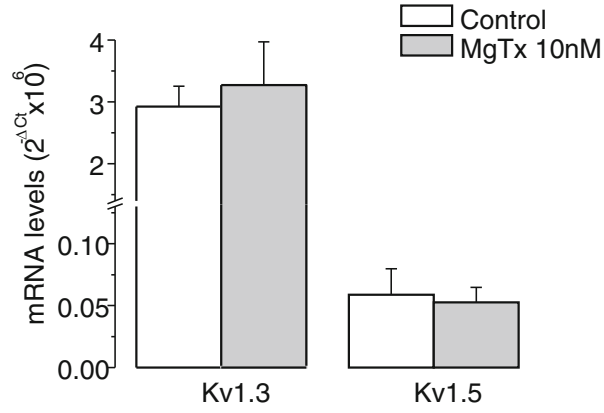

and $\mathrm{BPH}$ cultures. Data are mean $\pm \mathrm{SEM}$ of $4-8$ independent experiments. b The relative abundance of mRNA for Kv1.3 and Kv1.5 (normalized to the housekeeping gene 18S) in cultured VSMCs was not modify by the $24 \mathrm{~h}$ treatment with MgTx. The expression levels of both channels after $24 \mathrm{~h}$ incubation in $5 \% \mathrm{FBS}$ alone (control) or in the presence of $10 \mathrm{nM}$ $\operatorname{MgTx}$ are plotted. Mean $\pm \mathrm{SEM}, n=3$ experiments in duplicate determinations

Regarding our first conclusion, we found that hypertension induces a negative remodeling of the vessel wall in response to arterial injury that leads to an increased stenosis in our murine model of intimal hyperplasia. When characterizing the differences in the ion channel expression profile between injured BPH and injured BPN arteries, we observed that the enhanced VSMCs proliferation in injured BPH arteries correlates with an increased Kv1.3 to Kv1.5 ratio, an index that we have described associated with the phenotypic switch [13].

Hypertension and $\mathrm{K}^{+}$Channels Expression

$\mathrm{K}^{+}$channels have a wide spectrum of functions in the plasma membrane, and are important determinants of the cell

b

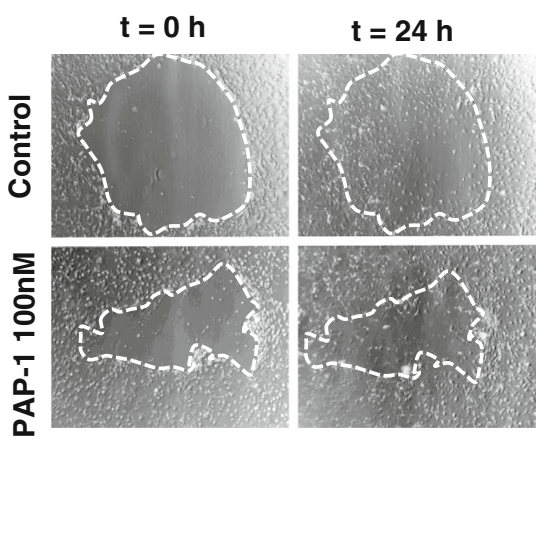

the presence of the Kv1.3 selective blocker PAP-1 (100 nM). Mean \pm SEM, $n=4$ experiments in different cultures. b The images show a representative experiment with $100 \mathrm{nM}$ PAP- 1 . The scratched area at $t=$ 0 is delimited with a dashed line, and the same line is superimposed in the images at $t=24 \mathrm{~h}$ to observe the migration 
Fig. 7 Effect of PAP-1 on intimal hyperplasia development after arterial injury. a

Representative microphotographs of femoral arteries of shamoperated BPN mice (upper panel), or 4 weeks after endoluminal injury upon treatment with either vehicle (middle panel) or PAP-1 (lower panel). b Bar-graphs show the morphometric analysis data; percentage of luminal stenosis and intima/media ratio

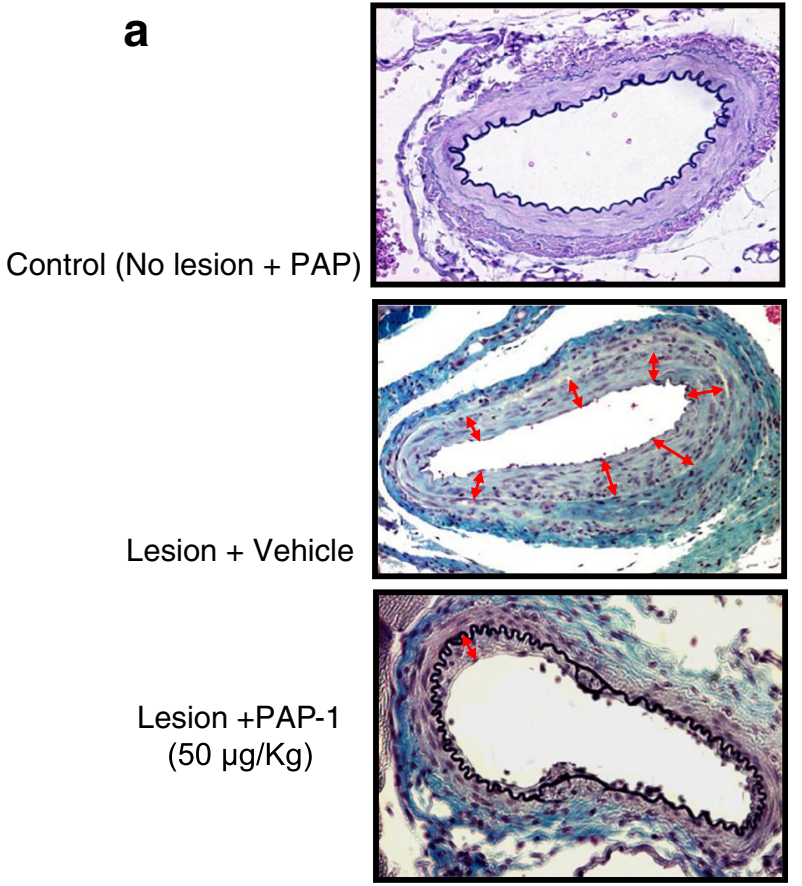

b
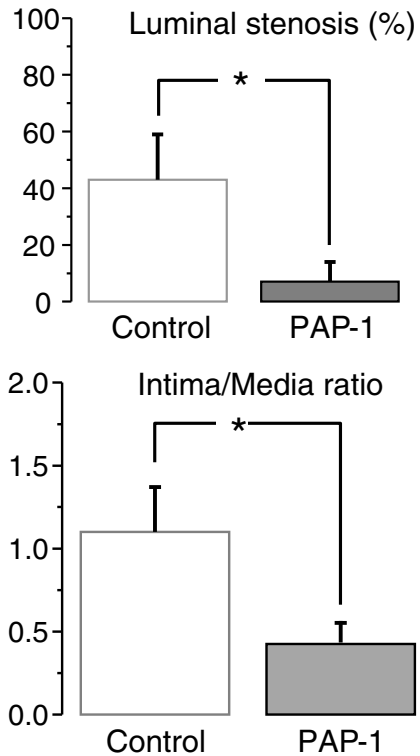

membrane potential. Changes in $\mathrm{K}^{+}$channels expression lead to changes in the vascular tone or cell phenotype, so that altered expression of potassium channels has been related to diseases such as systemic and pulmonary hypertension, atherosclerosis, and diabetes, among others $[18,19]$. In the present work, when comparing BPN and BPH animals, we detected several changes in $\mathrm{K}^{+}$channels expression that have been already described in some of these pathologies, such as the down regulation of BKCa channels, the decrease of Kv1.5 channels and the changes in the expression of inward-rectifier channels $[2,16,19]$. However, the most dramatic change, the up regulation of TWIK channels, could not be confirmed at the protein level, so that its possible functional contribution to the hypertensive phenotype awaits further characterization.

Of all the other observed changes, the decreased expression of Kv1.5 in hypertensive VSMCs both in control conditions and upon proliferation is especially relevant. Our data show that Kv1.5 is the most abundant $\mathrm{Kv}$ channel gene expressed in femoral VSMC in the contractile phenotype and it is also the predominant $\mathrm{Kv}$ current in these cells [2]. However, its expression decreases dramatically in proliferating VSMCs, where Kv1.5 current is almost non-detectable. In these cells, the larger $\mathrm{Kv}$ current seems to be carried by Kv1.3 channels [13]. These observations led us to postulate that the phenotypic switch of VSMCs associates with a Kv channel switch, from Kv1.5 in contractile VSMCs to Kv1.3 in proliferative VSMCs, so that Kv1.3 to Kv1.5 ratio could be a hallmark to define the phenotype. The data obtained in the present work when comparing proliferating VSMCs from BPN and BPH are consistent with this hypothesis, as the enhanced proliferation of BPH VSMCs associates with a larger Kv1.3 to Kv1.5 ratio. On the basis of this observation it is tempting to speculate that not only $\mathrm{Kv} 1.3 / \mathrm{Kv} 1.5$ ratio may serve as a tool to define VSMCs phenotype but also that changes in this ratio will modify the proliferative and migratory capabilities of the cells. More research in this line, by determining the effects of manipulating the expression of Kv1.3 and Kv1.5 on VSMCs phenotype will be required to confirm this attractive hypothesis.

\section{Hypertension and Intimal Hyperplasia}

Hypertension is a well-known risk factor for atherosclerosis development. In the clinical context, several factors concomitantly promote development of atherosclerosis, such as dyslipidemia, diabetes, smoking, obesity, hypertension, and age. Hypertension has been related to increased intimal thickening of the vascular wall in clinical and experimental models [20-22]. Among the mechanisms hypothesized for this effect is the increased expression of growth factors in hypertension and enhanced SMC proliferation in the vessel wall.

The interest of our intimal hyperplasia model resides in its similarity to human endoluminal procedures, with complete denudation and stretching of the vessel wall, due to guidewire passage. Using this model, we have observed a decreased vascular and luminal area in hypertensive animals, with similar intimal areas and intima-to-media ratios, resulting in a higher degree of luminal stenosis. These findings suggest, a negative remodeling, possibly due to increased vascular tone related to hypertension.

Compared with previously described models, like the carotid endarterectomy [22], our model resembles more closely 
the human setting after percutaneous coronary or vascular peripheral interventions. It is plausible that a mouse model of combined hyperlipidemia and hypertension, or older age, etc., like in the human clinical scenario, would have resulted in enhanced intimal hyperplasia in hypertensive animals and more marked differences in response-to-injury.

\section{Selective Blockade of Kv1.3 with PAP-1 Inhibits Intimal Hyperplasia}

We have previously described the predominant role of Kv1.3 in the process of phenotypic modulation and VSMC proliferation [13]. Therefore, inhibition of Kv1.3 channels could be a suitable therapeutic target to prevent intimal hyperplasia formation after vascular injury.

Kv1.3 channel blockers first clinical application has been as immunomodulators in T-cell mediated autoimmune diseases, such as diabetes, psoriasis, rheumatoid arthritis, multiple sclerosis and transplant rejection. Among all the compounds known to inhibit Kv1.3 channels, PAP-1 has the highest selectivity and is currently being under development for clinical use.

In the present study, we demonstrate that PAP-1 treatment in vivo inhibits intimal hyperplasia in a murine model of endoluminal arterial injury. Our data provide the first evidence of the antiproliferative effect of Kv1.3 blockers in an in vivo model, paving the way for the development of new therapies against stenosis directed to treat the root causes of the disease.

An antiproliferative and antimigratory effect of PAP-1, mediated by $\mathrm{Ca}^{2+}$ signaling inhibition, has been described on T-cells, which is linked to its immunosuppressive effect [12]. Also, selective inhibition of Kv1.3 has been shown to inhibit exocytosis of cytoplasmic granules from $\mathrm{CD} 4{ }^{+} \mathrm{CD} 28^{\text {null }} \mathrm{T}$ cells, which decreases atherosclerosis progression [23]. The present study is the first report, to date, of an antiproliferative and antimigratory effect of PAP-1 on vascular lineage cells in an in vivo model of endoluminal arterial injury. Kv1.3 up-regulation in proliferating VSMCs determines changes in resting membrane potential and/or intracellular $\left[\mathrm{Ca}^{2+}\right]$ that may promote proliferation [13]. In addition to the antiproliferative effect of Kv1.3 blockade in VSMCs, in the in vivo model the inhibitory effects of PAP-1 on $\mathrm{T}$ cells, platelets and macrophages are likely to have an important impact inhibiting both, atherosclerosis development, as well as intimal hyperplasia.

\section{Conclusions}

The results of our work show that hypertension led to a higher degree of luminal stenosis in our arterial injury model, that correlated with a decreased expression of Kv1.5 channels.
Also, our data confirm the role of Kv1.3 in intimal hyperplasia and the potential use of specific blocking agents, such as PAP1 , as a new therapeutic strategy to prevent restenosis after percutaneous coronary interventions.

\section{Future Directions}

Future investigations on the effect of the local administration of PAP-1 after coronary angioplasty and stent implantation in a superior animal model of restenosis is warranted, in order to assess efficacy and feasibility of this therapeutic strategy in a clinically relevant setting.

Acknowledgments We are grateful to Nadia Castillo and Esperanza Alonso for technical assistance in histological analysis and cell cultures.

Funding This work was funded by the Ministerio de Economia y Competitividad, Instituto de Salud Carlos III, (RIC RD12/0042/0006, Red Heracles) to MR, MH, MTP and JRL; FIS-Instituto Carlos III, PI11/00225 to MR, VALTEC 09-1-0042 to MR, a grant from the Spanish Heart Association to MR., Ministerio de Ciencia e Innovación grant BFU2010-15898 to MTPG and, Junta de Castilla y León grant VA094A11-2 to, JRLL

Disclosures None

\section{References}

1. Cox RH. Changes in the expression and function of arterial potassium channels during hypertension. Vasc Pharmacol. 2002;38:13-23.

2. Cidad P, Moreno-Domínguez A, Novensà L, et al. Characterization of ion channels involved in the proliferative response of femoral artery smooth muscle cells. Arterioscler Thromb Vasc Biol. 2010;6: 1203-11.

3. Friese RS, Mahboubi P, Mahapatra NR, et al. Am J Hypertens. 2005; 18:633.

4. Wulff H, Castle NA, Pardo LA. Voltage-gated potassium channels as therapeutic targets. Nat Rev Drug Discov. 2009;8:982-1001.

5. Kumar B, Dreja K, Shah SS, et al. Upregulated TRPC1 channel in vascular injury in vivo and its role in human neointimal hyperplasia. Circ Res. 2006;98:557-63.

6. Rodman DM, Harral J, Wu S, et al. Low-voltage-activated (T-type) calcium channels control proliferation of human pulmonary artery myocytes. Circ Res. 2005;96:864-72.

7. Miguel-Velado E, Pérez-Carretero FD, Colinas O, et al. Cell-cycle dependent expression of Kv3.4 channels modulates proliferation of human uterine artery smooth muscle cells. Cardiovasc Res 2010:86: 383-91.

8. Miguel-Velado E, Moreno-Domínguez A, Colinas O, et al. Contribution of $\mathrm{Kv}$ channels to phenotypic remodeling of human uterine artery smooth muscle cells. Circ Res. 2005;97:1280-7.

9. Tharp DL, Wamhoff BR, Wulff H, Raman G, Cheong A, Bowles DK. Local delivery of the KCa3.1 blocker, TRAM-34, prevents acute angioplasty-induced coronary smooth muscle phenotypic modulation and limits stenosis. Arterioscler Thromb Vasc Biol. 2008;28: 1084-9. 
10. Kohler R, Wulff H, Eichler I, et al. Blockade of the intermediateconductance calcium-activated potassium channel as a new therapeutic strategy for restenosis. Circulation. 2003;108:1119-25.

11. Villalonga N, David M, Bielanska J, et al. Immunomodulation of voltage-dependent $\mathrm{K}+$ channels in macrophages: molecular and biophysical consequences. J Gen Physiol. 2010;135:135-47.

12. Pardo LA. Voltage-gated potassium channels in cell proliferation. Physiology. 2004;19:285-92.

13. Cidad P, Jiménez-Pérez L, García-Arribas D, et al. Kv1.3 channels can modulate cell proliferation during phenotypic switch by an ionflux independent mechanism. Arterioscler Thromb Vasc Biol. 2012;32:1299-307.

14. Roque M, Fallon JT, Badimon JJ, Zhang WX, Taubman MB, Reis ED. Mouse model of femoral artery denudation injury associated with the rapid accumulation of adhesion molecules on the luminal surface and recruitment of neutrophils. Arterioscler Thromb Vasc Biol. 2000;20:335-42.

15. Schmitz A, Sankaranarayanan A, Azam P, et al. Design of PAP-1, a selective small molecule Kv1.3 blocker, for the suppression of effector memory $\mathrm{T}$ cells in autoimmune diseases. Mol Pharmacol. 2005;68:1254-70.

16. Moreno-Dominguez A, Cidad P, Miguel-Velado E, López-López JR, Pérez-García MT. De novo expression of Kv6.3 contributes to changes in vascular smooth muscle cell excitability in a hypertensive mice strain. J Physiol. 2009;587:625-40.

17. Livak KJ, Schmittgen TD. Analysis of relative gene expression data using real-time quantitative PCR and the 2(-Delta Delta C(T)) Method. Methods. 2001;25(4):402-8.

18. Yuan JX, Aldinger AM, Juhaszova M, et al. Dysfunctional voltagegated $\mathrm{K}+$ chanels in pulmonary artery smooth muscle cells of patients with primary pulmonary hypertension. Circulation. 1998;98:1400-6.

19. Korovkina VP, England SK. Detection and implications of potassium channel alterations. Vasc Pharmacol. 2002;38:3-12.

20. Cizek SM, Bedri S, Talusan P, Silva N, Lee H, Stone JR. Risk factors for atherosclerosis and the development of preatherosclerotic intimal hyperplasia. Cardiovasc Pathol. 2007;16:344-50.

21. Schindler R, Tullius SG, Tanriver Y, et al. Hypertension increases expression of growth factors and MHCII in chronic allograft nephropathy. Kidney Int. 2003;63:2302-8.

22. Drouilhet JC, Southern F, Williams KK, Brown AT, Eidt J, Moursi MM. Increased intimal hyperplasia after carotid endarterectomy in spontaneously hypertensive rats. Vasc Surg. 2001;35:11-8.

23. Wu X, Xu R, Cao M, Ruan L, Wang X, Zhang C. Effect of the Kv1.3 voltage-gated potassium channel blocker PAP-1 on the initiation and progress of atherosclerosis in a rat model. Heart Vessel 2014; Jan 19 (Epub ahead of print). 